Pacific Journal of Mathematics

RETICULATED SETS AND THE ISOMORPHISM OF ANALYTIC 


\title{
RETICULATED SETS AND THE ISOMORPHISM OF ANALYTIC POWERS
}

\author{
R. M. SHORTT
}

We study the properties of separable measurable spaces which are "Borel-dense of order $n$." Those Borel-dense of order 1 are precisely those that embed as a subset of the unit interval with totally imperfect complement, and the $n$th order version is an appropriate casting of this idea into $n$ dimensions. The concept enables one to sharpen some known results concerning the isomorphism types of analytic spaces. A result of Mauldin and Shortt (separately) may be stated thus:

(1) If $X$ is a space Borel-dense of order 1 and is Borel-isomorphic with $X \times X$, then $X$ is automatically a standard (absolute Borel) space. (Mauldin assumed $X$ to be analytic.)

We obtain the following enlargement:

(2) If $X$ is a space Borel-dense of order $n$ and $X^{n}$ is Borel-isomorphic with $X^{m}$ (some $m>n$ ), then $X$ is an analytic space.

The requirement of $n$th order density is not overly severe. Complements (in a standard space) of universally null sets are Borel-dense of every finite order, for example; the same may be said for complements of sets always of first category or, more generally, of sets with Marczewski's property $\left(s^{0}\right)$. Statement 2 might therefore be regarded as a criterion whereby to judge which universally null sets (or sets always of first category, or sets with property $\left(s^{0}\right)$ ) are co-analytic. It should also be mentioned, however, that the problem of finding a particular Borel-dense non-Borel analytic space $A$ for which $A^{2} \cong A^{3}$ is open; it may be that "analytic" in statement 2 can be strengthened to "standard". The relationship between Borel-density and the Blackwell property is also noted.

Our method of proof revolves around a strengthening of a classical theorem of Mazurkiewicz and Sierpiński [10] to the effect that if $A$ is an analytic subset of a product $S_{1} \times S_{2}$, then the set of $s$ in $S_{1}$ such that the section $A(s)$ is uncountable is analytic. A multi-dimensional version of this theorem is proposition 1 infra, wherein "uncountable" is replaced with "non-recticulate" in keeping with the dimensions of the sections. The fact that the projection of an analytic set is again analytic is expanded into this multi-dimensional setting in Proposition 2. Other classical results of Lusin and Braun do not generalize, however, as is shown by an example. 
1. Notations, preliminaries. We work exclusively with separable spaces, i.e. measurable spaces $(X, \mathscr{B})$ with a countably generated and separated $\sigma$-algebra $\mathscr{B}$. Often, the notation of the $\sigma$-algebra is suppressed. If $A$ is a subset of $X$, then $A$ is considered a separable space under its relative structure $\mathscr{B}(A)=\{B \cap A: B \in \mathscr{B}\}$. We assume that the reader is familiar with the elements of descriptive set theory and the study of Borel spaces, in particular the theory of standard and analytic spaces. As references, we give Kuratowski [6], Hoffmann-Jørgensen [5], and Rao and Bhaskara Rao [11].

In much of what follows, we shall be considering a collection of (usually uncountable standard) spaces $S_{1}, \ldots, S_{n}$ and subsets $A$ of their product $S=S_{1} \times \cdots \times S_{n}$. By a $k$-slice of $S$ we mean a set of the form $A_{1} \times \cdots \times A_{n}$, where $k$ of these factor sets are singletons and the other $n-k$ sets $A_{j}$ are equal to $S_{j}$. An $n$-slice of $S$ is thus a single point of $S$, whilst the only 0 -slice is $S$ itself. If $\left\{i_{1}, \ldots, i_{k}\right\}$ is a subset of $\{1, \ldots, n\}$ of cardinality $k$, and $s_{1}, \ldots, s_{k}$ are elements of $S_{i_{1}}, \ldots, S_{i_{k}}$, respectively, then $\left\{\bar{s} \in S: \bar{s}\left(i_{j}\right)=s_{j}, j=1, \ldots, k\right\}$ is the $k$-slice of $S$ over the point $\left(s_{1}, \ldots, s_{k}\right)$; it will occasionally be identified with its projection on $\Pi\left\{S_{i}: i \neq i_{J}\right.$; $j=1, \ldots, k\}$.

If $A \subset S$, then by a $k$-section of $A$ we mean the intersection of $A$ with a $k$-slice of $S$; if $B$ is a $k$-slice of $S$ over the point $\left(s_{1}, \ldots, s_{k}\right)$, then $B \cap A$ is the $k$-section of $A$ over the point $\left(s_{1}, \ldots, s_{k}\right)$ and is denoted $A\left(s_{1}, \ldots, s_{k}\right)$. Again, these sets are sometimes identified with their projections onto the corresponding $(n-k)$-dimensional partial product. If $k$ is an integer $0 \leq k \leq n$, then $\|A\|_{k}$ is the smallest cardinality of a collection of $k$-sections of $S$ whose union contains $A$ as a subset. Obviously, $\|A\|_{0} \leq\|A\|_{1} \leq$ $\cdots \leq\|A\|_{n} ;\|A\|_{n}$ denotes the cardinality of $A$, whereas $\|A\|_{0}$ is zero or unity according as $A$ is null or non-void. A set $A$ is $k$-reticulate in $S$ if it is contained in some countable union of $k$-slices of $S$, i.e. if $\|A\|_{k} \leq \boldsymbol{\aleph}_{0}$. In $S=S_{1} \times \cdots \times S_{n}$, the terms " $n$-reticulate" and "countable" are synonymous, while every subset of $S$ is 0 -reticulate. By a thread of $S$ is meant an uncountable standard subset of $S$, each of whose 1-sections contains at most one point.

Let $S_{1}, \ldots, S_{n}$ be standard spaces and let $X_{1} \subset S_{1}, \ldots, X_{n} \subset S_{n}$ be subsets of these. Say that $X_{1}, \ldots, X_{n}$ are jointly Borel-dense of order $n$ in $S_{1}, \ldots, S_{n}$ if every analytic subset $A$ of $\left(S_{1} \times \cdots \times S_{n}\right) \backslash\left(X_{1} \times \cdots \times X_{n}\right)$ is contained in a countable union of 1-slices of $S_{1} \times \cdots \times S_{n}$ over points in $S_{j} \backslash X_{j}, j=1, \ldots, n$. A separable space $X$ is Borel-dense of order $n$ if $X$ embeds as a subset of a standard space $S$ in such a way that $X, \ldots, X$ ( $n$-times) are jointly Borel-dense of order $n$ in $S, \ldots, S$ ( $n$-times). 
The notion of Borel-density was studied in Shortt [12] and [13], and we summarize a few of the main results presently:

LEMMA 1. If a separable space $X$ can be written as a countable union $X=X_{1} \cup X_{2} \cup \cdots$ of sets $X_{i}$, each Borel-dense of order 1, then $X$ is Borel-dense of order 1.

Proof. Embed $X$ in some standard space $S$. Then there are sets $S_{1}, S_{2}, \ldots$, in $\mathscr{B}(S)$ with $X_{i} \subset S_{\imath}$ and $S_{\iota} \backslash X_{i}$ totally imperfect. $S_{0}=S_{1} \cup$ $S_{2} \cup \cdots$ is standard, contains $X$, and is such that $S_{0} \backslash X$ is totally imperfect.

LEMMA 2. If a separable space $X$ is Borel-dense of order 1, then so is any member of $\mathscr{B}(X)$.

Proof. Embed $X$ in a standard space $S$ with $S \backslash X$ totally imperfect. Then each $A$ in $\mathscr{B}(X)$ may be written as $B \cap X$ for some $B$ in $\mathscr{B}(X)$. Since $B \backslash A \subset S \backslash X$, the lemma follows.

LEMMA 3. (1) if $X$ is a universally measurable space (resp. space with the restricted Baire property, resp. space with property $(s))$ which is Borel-dense of order 1, then $X$ is Borel-dense of order $n=1,2, \ldots$ (Such spaces are precisely the complements of universally null sets (resp. sets always of first category, resp. sets with property $\left(s^{0}\right)$ ) in a standard space.)

(2) If $X$ is Borel-dense of order 1, then $X$ has the Blackwell property if and only if $X$ is Borel-dense of order 2. (For such spaces, strong and weak Blackwell properties are equivalent.)

(3) If $X_{1}$ and $X_{2}$ are uncountable separable spaces such that $X_{1} \times X_{2}$ is Borel-dense of order 1 , then $X_{1}$ and $X_{2}$ are standard.

Proof. (1) Corollary 5 in Shortt [12] gives an argument for universally measurable spaces that generalizes straightforwardly to the other cases.

(2) This is the principal result in Shortt [13].

(3) Proposition 13 in Shortt [12]; compare also Mauldin [9], Remark 4 in Grzegorek and Ryll-Nardzewski [3], and a new proof to appear in Grzegorek [4].

The following fact will be in frequent employ throughout the sequel:

Lemma 4. Let $A_{1}$ and $A_{2}$ be subsets of the standard spaces $S_{1}$ and $S_{2}$, respectively. Suppose that $g$ is a Borel-isomorphism of $A_{1}$ onto $A_{2}$. If $S_{1} \backslash A_{1}$ and $S_{2} \backslash A_{2}$ contain uncountable Borel subsets of $S_{1}$ and $S_{2}$, then $g$ extends to an isomorphism of $S_{1}$ onto $S_{2}$. 
Proof. Apply the extension theorem in Kuratowski [6, §36, VII] and the fact that any two uncountable standard spaces are Borel-isomorphic.

To conclude this preliminary section, we present some results on subsets of a product space containing a "thread":

LemMA 5. Let $S_{1}, \ldots, S_{n}$ be standard spaces and let $A$ be an uncountable analytic subset of $S=S_{1} \times \cdots \times S_{n}$, each of whose 1-sections is countable. Then A contains a thread.

Proof. A selection theorem of Lusin [7, p. 243] (also see HoffmannJørgensen [5, III.6.7]) implies that there is an uncountable analytic $A_{1} \subset A$ which is the graph of a one-one measurable function from an analytic subset of $S_{1}$ onto an (analytic) subset of $S_{2} \times \cdots \times S_{n}$. Repeating this argument, we obtain uncountable analytic sets $A \supset A_{1} \supset A_{2} \supset \cdots \supset A_{n}$ such that $A_{i}$ is the graph of a one-one measurable function from some analytic subset of $S_{i}$ onto a subset of $S_{1} \times \cdots \times S_{i-1} \times S_{i+1} \times \cdots \times S_{n}$. Every non-empty 1 -section of $A_{n}$ is a singleton set. Any uncountable standard subset of $A_{n}$ is a thread.

Lemma 6. Let $S_{1}, \ldots, S_{n}$ and $P$ be Polish spaces. Suppose that $f$ : $P \rightarrow S_{1} \times \cdots \times S_{n}$ is a continuous function whose image $A=f(P)$ is an (analytic) subset of $S=S_{1} \times \cdots \times S_{n}$. Then amongst the following three statements, (1) implies (2), and (2) implies (3):

(1) A contains a thread of $S$.

(2) There is a dense-in-itself sequence of points of $P$ on which each of the component functions $f_{1}, \ldots, f_{n}$ of $f$ is one-one.

(3) $A$ is not 1-reticulate in $S$.

Proof. (1) implies (2): Suppose that $T \subset A$ is a thread of $S$. Then $f$ is continuous from $f^{-1}(T)$ onto $T$, and the implication follows from Kuratowski [6, §36, V].

(2) implies (3): We assume that $E \subset P$ is such a countable dense-in-itself set and look for a contradiction. Suppose that $A$ is contained in $C_{1} \cup C_{2} \cup \cdots$, where each $C_{j}$ is some 1-slice of $S$. Put $X_{j}=f^{-1}\left(C_{j}\right)$ for $j=1,2, \ldots$ Then $P=X_{1} \cup X_{2} \cup \cdots$ expresses $P$ as the union of a sequence of closed sets. Since each of the components $f_{1}, \ldots, f_{n}$ is one-one on $E$, it follows that, for each $j, E \cap X_{j}$ contains at most one point and is therefore scattered. From Kuratowski [6, §34, IV, Corollary 5], $E$ is scattered, a contradiction. 
Conjecture. The three conditions in Lemma 6 are actually equivalent.

Recent work of Graf and Mauldin [2, Theorem 4.4] shows this to be true when $n=2$. The case $n=\infty$ might also be of interest.

LEMMA 7. Let $S_{1}, \ldots, S_{n}$ be standard spaces and suppose that $A$ is an analytic subset of the product $S=S_{1} \times \cdots \times S_{n}$. Then $A$ is $k$-reticulate in $S(1 \leq k \leq n)$ if and only if these three conditions are satisfied:

(1) Each 1-section of $A$ is $(k-1)$-reticulate. (This condition is vacuous if $k=1$.)

(2) Given a subset $F=\left\{i_{1}, \ldots, i_{r}\right\} \subset\{1, \ldots, n\}$ of cardinality $r, k \leq r$ $\leq n$, define the sets

$$
\begin{aligned}
& A(F)=\left\{\left(s_{i_{1}}, \ldots, s_{i r}\right) \in S_{i_{1}} \times \cdots \times S_{i_{r}}:\right. \\
& \left.A\left(s_{i_{1}}, \ldots, s_{i_{r}}\right) \text { is not 1-reticulate }\right\} .
\end{aligned}
$$

Each of these sets $A(F)$ is $k$-reticulate.

(3) $A$ contains no thread of $S$.

Proof. The necessity of the three conditions is easily verified, if a little cumbrous to write out. To prove sufficiency, note first that condition (2) implies that by removing countably many $k$-slices of $S$ from $A$, one may obtain a set $A^{\prime}$ each of whose $r$-sections, $k \leq r \leq n$, is 1-reticulate. In particular, each $(n-1)$-section of $A^{\prime}$ is countable, and each $(n-2)$ section of $A^{\prime}$ is contained in a countable union of 1 -slices. It follows that each $(n-2)$-section of $A^{\prime}$ is countable. Proceeding in iterative fashion, we see that each $k$-section of $A^{\prime}$ is countable.

Claim. Each 1-section of $A^{\prime}$ is countable. If $k=1$, this has already been shown. For $k>1$, we use condition 1: each 1-section of $A$ and hence of $A^{\prime}$ is $(k-1)$-reticulate. Because each $k$-section of $A^{\prime}$ is countable, the claim is seen to be proved.

Now $A^{\prime}$ was formed from $A$ by the removal of countably many 1-sections. Lemma 5 and condition 3 combine to show that $A^{\prime}$ is in fact countable. It follows that $A$ is $k$-reticulate.

In addition to the role it plays in the development of the next section, this lemma enables us to establish a special continuum hypothesis for the cardinalities $\|A\|_{k}$ as follows:

LEMMA 8. Let $S_{1}, \ldots, S_{n}$ be standard spaces and suppose that $A$ is an analytic subset of the product $S=S_{1} \times \cdots \times S_{n}$. If $\|A\|_{k}<c$, then $\|A\|_{k}$ $\leq \boldsymbol{\aleph}_{0}$, i.e. $A$ is $k$-reticulate in $S$. 
Proof. We proceed via induction on the dimension $n$, noting that the case $k=n$ (in particular $n=1$ ) is subsumed by the special continuum hypothesis for analytic sets. So assume that $1 \leq k \leq n$ and that the lemma obtains in dimensions $1, \ldots, n-1$.

It remains only to verify the three conditions set forth in Lemma 7. Each 1-section of $A$ is contained in fewer than $c(k-1)$-sections and so from the induction hypothesis is $(k-1)$-reticulate. A similar reasoning establishes condition 2: note that the pairs

$$
\left\{\begin{array} { l } 
{ \| A ( s _ { i _ { 1 } } , \ldots , S _ { i _ { r } } ) \| _ { k } < c } \\
{ \| A ( s _ { i _ { 1 } } , \ldots , s _ { i _ { r } } ) \| _ { k } \leq \boldsymbol { \aleph } _ { 0 } }
\end{array} \text { and } \left\{\begin{array}{l}
\|A(F)\|_{k}<c \\
\|A(F)\|_{k} \leq \boldsymbol{\aleph}_{0}
\end{array}\right.\right.
$$

are, by the induction hypothesis, equivalent. Condition 3 is immediate.

We conclude this section with a combinatorial result to be used in the next section. Its proof is perhaps of some independent interest.

LEMMA 9. Let $S_{1}, \ldots, S_{n}$ be arbitrary non-empty sets and let $A$ be a subset of the product $S=S_{1} \times \cdots \times S_{n}$. Let $m$ and $k$ be non-negative integers, $0 \leq k \leq n$. Then $\|A\|_{k} \geq m$ if and only if there is a finite subset $P$ of $A$ with $\|P\|_{k} \geq m$.

Proof. Endow the sets $S_{1}, \ldots, S_{n}$ with compact Hausdorff topologies (there is such a topology on every set) and give $S$ the corresponding product topology. Let $\mathscr{K}$ be the hyper-space of all closed subsets of $S$ under the exponential topology of Hausdorff and Vietoris (v. Kuratowski $[6, \S 17])$ and let $\mathscr{K}(m)$ be the sub-space of all unions of fewer than $m$ $k$-slices of $S$. Since the map sending a point $s=\left(s_{1}, \ldots, s_{k}\right)$ to the $k$-slice over $s$ is continuous into $\mathscr{K}$, and because the operation of union is continuous, it follows that $\mathscr{K}(m)$ is compact. ( $\mathscr{K}$ is also compact, but this will not be needed.)

Now let $\kappa$ be the least cardinal number for which there is a subset $P$ of $A$ or cardinality $\kappa$ such that $\|P\|_{k} \geq m$. It suffices to prove that $\kappa$ is finite. Supposing contrariwise that $\kappa$ is infinite and employing a wellordering, one may write $P$ as an ascending union $P_{0} \subset P_{1} \subset \cdots \subset P_{\alpha} \subset$ $\cdots$ of sets indexed by all ordinals $\alpha<\kappa$, with each $P_{\alpha}$ of power less than $\kappa$. For each $\alpha<\kappa$, let $K_{\alpha}$ be a member of $\mathscr{K}(m)$ with $P_{\alpha} \subset K_{\alpha}$. By the compactness of $\mathscr{K}(m)$, there is a sub-net $\left\{K_{\alpha(\lambda)}\right\}_{\lambda}$ of $\left\{K_{\alpha}\right\}_{\alpha}$ converging to some $K$ in $\mathscr{K}(m)$. It follows that $K$ contains each $P_{\alpha}$ and so $P \subset K$, a contradiction.

2. Reticulate sections. We begin by recalling the classical theorem of Mazurkiewicz and Sierpiński [10] on sections of an analytic set. 
THEOREM (Mazurkiewicz-Sierpinski). Let $A$ be an analytic subset of the product $S_{1} \times S_{2}$ of standard spaces $S_{1}$ and $S_{2}$. Then the set $\left\{s \in S_{1}: A(s)\right.$ is uncountable $\}$ is analytic.

Proof. In addition to the original 1924 paper, one might also consult Kuratowski [6, §39, VII] and Hoffmann-Jørgensen [5, III.6.1].

The following generalization of this theorem will serve as our primary tool for investigation of product isomorphisms.

Proposition 1. Let $S_{0}, S_{1}, \ldots, S_{n}$ be Polish spaces and let $B$ be an analytic subset of $S=S_{0} \times S_{1} \times \cdots \times S_{n}$. For each integer $k, 1 \leq k \leq n$, the set

$$
A=\left\{s \in S_{0}: B(s) \text { is not k-reticulate in } S_{1} \times \cdots \times S_{n}\right\}
$$

is an analytic subset of $S_{0}$.

Demonstration. We proceed by induction on the dimension $n$, noting that the case $k=n$ (in particular $n=1$ ) is the classical MazurkiewiczSierpinski Theorem. So assume that $1 \leq k<n$ and that the proposition is true in dimensions, $1,2, \ldots, n-1$.

In the case where $k=1$, define $A_{0}$ to be the null set. If $k>1$, then for each $i=1, \ldots, n$, define

$$
B_{\imath}=\left\{\left(s, s_{i}\right) \in S_{0} \times S_{i}: B\left(s, s_{\imath}\right) \text { is not }(k-1) \text {-reticulate }\right\}
$$

and let $A_{0}$ be the union of the projections of the $B_{i}$ onto $S_{0}$. By the induction hypothesis, each $B_{i}$ (and hence $A_{0}$ ) is analytic.

Given a subset $F=\left\{i_{1}, \ldots, i_{m}\right\}$ of $\{1, \ldots, n\}$ of cardinality $m, k \leq m$ $<n$, define the sets

$$
\begin{aligned}
& B(F)=\left\{\left(s, s_{i_{1}}, \ldots, s_{i_{m}}\right) \in S_{0} \times S_{i_{1}} \times \cdots \times S_{l_{m}}:\right. \\
& \left.B\left(s, s_{i_{1}}, \ldots, s_{l_{m}}\right) \text { is not 1-reticulate }\right\}
\end{aligned}
$$

and

$$
A(F)=\left\{s \in S_{0}: B(F)(s) \text { is not } k \text {-reticulate }\right\} .
$$

Again using the induction hypothesis, we see that the sets $B(F)$ and $A(F)$ are analytic. For each $m, k \leq m<n$, define $A_{m}$ to be the union of all $A(F)$ as $F$ ranges over all subsets of $\{1, \ldots, n\}$ of cardinality $m$.

Since $B$ is analytic, there is a Polish space $P$ and a continuous function $f: P \rightarrow S$ mapping $P$ onto $B$. Let $f_{0}, f_{1}, \ldots, f_{n}$ be the components of the function $f$. Define $Z$ to be the (Polish) space of all sequences in 
$P^{\infty}=P \times P \times \cdots$ that are dense-in-themselves. Cf. Kuratowski [6, §30, XII]. Define $A_{n}$ to be the set of all $s$ in $S_{0}$ such, that there is a dense-in-itself sequence of points of $P$ on which:

(1) $f_{0}$ is identically equal to $s$, and

(2) each of the functions $f_{1}, \ldots, f_{n}$ is one-one. $A_{n}$ is the projection on $S_{0}$ of the following subset of $S_{0} \times Z$ :

$$
\begin{aligned}
\bigcap_{k=1}^{\infty}\left\{(s, z): s=f_{0}(z(k))\right\} \\
\quad \cap \bigcap_{i=1}^{n} \bigcap_{k \neq l}\left\{(s, z): f_{l}(z(k)) \neq f_{i}(z(l))\right\} .
\end{aligned}
$$

Since this last is a $G_{\delta}$ set, its projection $A_{n}$ is analytic.

Lemmas 6 and 7 imply that $A=A_{0} \cup A_{k} \cup A_{k+1} \cup \cdots \cup A_{n}$, which fact establishes the proposition.

Corollary. Let $S_{0}, S_{1}, \ldots, S_{n}$ be Polish spaces and let $f: D \rightarrow S_{0}$ be a measurable function defined on an analytic subset $D$ of $S_{1} \times \cdots \times S_{n}$. For each integer $k, 1 \leq k \leq n$, the set

$$
\left\{s \in S_{0}: f^{-1}(s) \text { is not } k \text {-reticulate in } S_{1} \times \cdots \times S_{n}\right\}
$$

is an analytic subset of $S_{0}$.

Proof. Since $f$ is measurable, the graph of $f$ is an analytic subset of $S_{0} \times S_{1} \times \cdots \times S_{n}$; the sets $f^{-1}(s)$ are sections of this graph over points $s$ in $S_{0}$. The previous theorem now applies.

The following result elaborates upon the theme that the projection of an analytic set is again analytic. Compare Hoffmann-Jørgensen [5, III.5.1].

Proposition 2. Let $A$ be an analytic subset of the product $S_{0} \times S_{1} \times$ $\cdots \times S_{n}$ of standard spaces. Let $m$ and $k$ be non-negative integers $0 \leq k \leq n$. Then the set

$$
A(m, k)=\left\{s \in S_{0}:\|A(s)\|_{k} \geq m\right\}
$$

is analytic.

Demonstration. Noting that $A(1, k)$ is merely the projection of $A$ onto $S_{0}$ and is therefore analytic, we may assume that $m \geq 2$; also, we take $k \geq 1$ ( $k=0$ is trivial). For each positive integer $l$, define $A_{l}$ to be the set of all $s$ in $S_{0}$ such, that $A(s)$ has a subset $P=\left\{p_{1}, \ldots, p_{l}\right\}$ of cardinality not exceeding $l$ for which $\|P\|_{k} \geq m$. With this notation, we do not mean that the $p_{i}$ 's are necessarily distinct. Such a set $P$ is not contained in any 
union of $m-1 k$-sections of $S=S_{1} \times \cdots \times S_{n}$. Otherwise put, let $\mathscr{N}$ be the collection of all $k$-element subsets of $\{1, \ldots, n\}$; then for any subset $\mathscr{F}$ of $\mathscr{N} \times\{1, \ldots, l\}$ of power $m-1$, there is some point $p_{r}$ in $P$ not contained in any of the $k$-sections of $S$ over the points $\left(p_{j}\left(i_{1}\right), \ldots, p_{j}\left(i_{k}\right)\right)$ for $\left\{i_{1}, \ldots, i_{k}\right\} \times\{j\} \in \mathscr{F}$. Here, $p_{j}(i)$ denotes the $i$ th co-ordinate of $p_{j}$. Thus, $\left(p_{j}\left(i_{1}\right), \ldots, p_{j}\left(i_{k}\right)\right) \neq\left(p_{r}\left(i_{1}\right), \ldots, p_{r}\left(i_{k}\right)\right)$ for each $\left\{i_{1}, \ldots, i_{k}\right\} \times\{j\}$ in $\mathscr{F}$.

It therefore becomes possible to write $A_{l}$ as the projection on $S_{0}$ of the following subset of $S_{0} \times S \times \cdots \times S$ :

$$
\begin{aligned}
& \bigcap_{\mathscr{F}} \bigcup_{r=1}^{l} \cap\left\{\left(s, p_{1}, \ldots, p_{l}\right):\left(s, p_{1}\right), \ldots,\left(s, p_{l}\right) \in A\right. \\
& \left.\quad \text { and }\left(p_{j}\left(i_{1}\right), \ldots, p_{j}\left(i_{k}\right)\right) \neq\left(p_{r}\left(i_{1}\right), \ldots, p_{r}\left(i_{k}\right)\right)\right\},
\end{aligned}
$$

where the index $\mathscr{F}$ ranges over all subsets of $\mathscr{N} \times\{1, \ldots, l\}$ of cardinality $m-1$ and where the second intersection is taken over all elements $\left\{i_{1}, \ldots, i_{k}\right\} \times\{j\}$ of $\mathscr{F}$. Thus, each $A_{l}$ is an analytic subset of $S_{0}$.

By Lemma 9, $A(m, k)=A_{m} \cup A_{m+1} \cup A_{m+2} \cup \cdots$, and so $A(m, k)$ is analytic.

COROLLARY. In the same content as the proposition, the sets

$$
A(\infty, k)=\left\{s \in S_{0}:\|A(s)\|_{k} \geq \boldsymbol{\aleph}_{0}\right\}
$$

are also analytic.

Proof. Immediate from the identity $A(\infty, k)=\bigcap_{m=1}^{\infty} A(m, k)$.

Corollary. Let $S_{0}, S_{1}, \ldots, S_{n}$ be standard spaces and let $f: D \rightarrow S_{0}$ be a measurable function defined on an analytic subset $D$ of $S_{1} \times \cdots \times S_{n}$. For each $k, 0 \leq k \leq n$, and $m \geq 0$, the set

$$
\left\{s \in S_{0}:\left\|f^{-1}(s)\right\|_{k} \geq m\right\}
$$

is an analytic subset of $S_{0}$.

Proof. Apply the preceding proposition to the graph of $f$ and its sections $f^{-1}(s)$.

A classical result of Lusin [7, p. 257] runs as follows:

Theorem (Lusin). Let $A$ be an analytic subset of the product $S_{1} \times S_{2}$ of standard spaces $S_{1}$ and $S_{2}$. Then the set $\left\{s \in S_{1}: A(s)\right.$ is singleton $\}$ is co-analytic. 
Generalizations and supplements for this theorem are to be found in Braun [1], where the phase "is singleton" is replaced by "contains an isolated point" and "is countable" \&c.. Unfortunately, the analogous results do not hold in the present context, at least not without some extra restrictions. We conclude this section with an example to illustrate the point.

EXAMPLE. Let $S_{1}$ and $S_{2}$ be standard spaces and let $B$ be a Borel subset of $S_{1} \times S_{2}$ whose projection onto $S_{1}$ is not Borel. Define $f: B \rightarrow S_{1}$ to be projection onto the first co-ordinate. Then $f(B)=\left\{s \in S_{1}\right.$ : $\left.\left\|f^{-1}(s)\right\|_{1}=1\right\}$ is not co-analytic.

A satisfactory generalization of some of Lusin and Braun's other results might yet be attempted; it may be impossible, but worth it nonetheless.

3. Isomorphism of powers. The concept of reticulation gives some insight into the structure of product spaces; in particular, it relates the regularity of certain Borel-dense spaces to their behavior under Cartesian multiplication. As long as Borel-dense spaces and their totally imperfect complements continue as objects of study, such results will have their place. For example, proposition 3 suggests that some sort of dimension theory might be developed for Borel-dense spaces.

We are now ready for our principal result on isomorphisms of product spaces. It is interesting to note that the full strength of proposition 1 , i.e. for $k=1, \ldots, n$, is used in the proof. By the notation $X^{m}$ is meant the $m$-fold product of the space $X$ with itself, with the convention that $X^{0}$ is a one-point space.

Proposition 3. Let $X$ be a separable space, Borel-dense of order $n$, where $n$ is a fixed positive integer. If $X^{n}$ is Borel-isomorphic with a product $X^{n-1} \times A_{n} \times A_{n+1}$, where $A_{n}$ and $A_{n+1}$ are uncountable separable spaces, then $X$ is analytic.

Demonstration. First note that for $n=1$, this is part 3 of Lemma 3: in this special case, it can be concluded that $X$ is a standard space. For the rest of the proof, we assume that $n \geq 2$.

Case I. Both of the spaces $A_{n}$ and $A_{n+1}$ are standard. Let $S$ be an uncountable standard space. In this case, $X^{n}$ and $X^{n-1} \times S \times S$ are isomorphic. Since $S$ and $S \times S$ are isomorphic, we have the isomorphisms 
$X^{n} \cong X^{n-1} \times S \cong X^{n-1} \times S \times S \cong X^{n-1} \times X \times S$. By taking $A_{n}^{\prime}=X$ and $A_{n+1}^{\prime}=S$, we see that case 1 reduces to

Case II. At least one of the spaces $A_{n}, A_{n+1}$ is not standard. We assume that $X$ is not analytic and derive a contradiction. Suppose that $X$ is Borel-dense of order $n$ in the (uncountable) standard space $S$ and that $g: X^{n} \rightarrow X^{n-1} \times A_{n} \times A_{n+1}$ is a Borel-isomorphism. By Lemma 4, $g$ extends to an isomorphism $f$ of $S^{n}$ onto a product of standard spaces $S_{1} \times S_{2} \times \cdots \times S_{n} \times S_{n+1}$. Let the components of $f$ be denoted by $f_{1}, f_{2}, \ldots, f_{n+1}$ and consider the set

$$
B_{1}=\left\{s \in S_{1}: f_{1}^{-1}(s) \text { is not 1-reticulate in } S^{n}\right\} \text {. }
$$

Since $X$ is Borel-dense of order $n$ in $S$, one has $B_{1} \subset X$. From the reticulation theorem, $B_{1}$ is analytic, and so there must be some $x_{1}$ in $X$ with $f_{1}^{-1}\left(x_{1}\right)$ 1-reticulate in $S^{n}$. Now consider the set

$$
B_{2}=\left\{s \in S_{2}: f_{1}^{-1}\left(x_{1}\right) \cap f_{2}^{-1}(s) \text { is not 2-reticulate in } S^{n}\right\} \text {. }
$$

The reticulation theorem, applied to the restriction of $f_{2}$ to $f_{1}^{-1}\left(\mathrm{x}_{1}\right)$, shows $B_{2}$ to be analytic. Given $s \in S_{2} \backslash X$, we see that $f_{1}^{-1}\left(x_{1}\right) \cap f_{2}^{-1}(s)$ is a 1-reticulate subset of $S^{n}$ contained in $S^{n} \backslash X^{n}$. Its intersection with any 1-slice of $S^{n}$ may therefore be considered a subset of $S^{n-1} \backslash X^{n-1}$. It follows from the $(n-1)$-order Borel-density of $X$ that $f_{1}^{-1}\left(x_{1}\right) \cap f_{2}^{-1}(s)$ is 2-reticulate in $S^{n}$. So $B_{2} \subset X$, and so there is some $x_{2}$ in $X$ with $f_{1}^{-1}\left(x_{1}\right) \cap f_{2}^{-1}\left(x_{2}\right)$ 2-reticulate in $S^{n}$.

This process continues until elements $x_{1}, \ldots, x_{n-1}$ of $X$ are produced with $f_{1}^{-1}\left(x_{1}\right) \cap \cdots \cap f_{n-1}^{-1}\left(x_{n-1}\right)$ an $(n-1)$-reticulate subset of $S^{n}$. Then, under the map $f^{-1}$, the space $\left\{x_{1}\right\} \times \cdots \times\left\{x_{n-1}\right\} \times A_{n} \times A_{n+1}$ and hence $A_{n} \times A_{n+1}$ is Borel-isomorphic with an $(n-1)$-reticulate Borel subset of $X^{n}$. But any $(n-1)$-reticulate Borel subset of $X^{n}$ is isomorphic with a countable union (in $S^{n}$ ) of Borel subsets of $X$ and so is Borel-dense. By part 3 of Lemma $3, A_{n}$ and $A_{n+1}$ must be standard, a contradiction.

Proposition 4. Let $X$ be a separable space, Borel-dense of order $n$. If $X^{n}$ is Borel-isomorphic with a 1-reticulate Borel subset of itself, then $X$ is analytic.

Demonstration. This is an application of the same method as was used in the proof of Proposition 3, Case II. The details are omitted.

Corollary. Let $X$ be Borel-dense of order $n$. If $X^{n}$ is Borel-isomorphic with the direct sum of a finite or countably infinite number of copies of $X^{n-1}$, then $X$ is analytic. 
Proof. Such a direct sum may be regarded as a 1-reticulate Borel subset of $X^{n}$. The preceding proposition now applies.

Numerous other sequelae of Propositions 3 and 4 could now be added, all similar in character to the foregoing corollary. This similarity precludes a listing here.

Added in proof. H. Sarbadhikari has established the conjecture mentioned after Lemma 6.

\section{REFERENCES}

[1] S. Braun, Quelques théorèmes sur les cribles boreliens, Fund. Math., 20 (1933), 166-176.

[2] S. Graf and R. D. Mauldin, Measurable one-to-one selections and transition kernels, Amer. J. Math., (1983), (to appear).

[3] E. Grzegorek and C. Ryll-Nardzewski, A remark on absolutely measurable sets, Bulletin de L'Acad. Polon. des Sci., XXVIII (1980), 229-232.

[4] E. Grzegorek, On Borel structures, Proceedings Oberwolfach Measure Theory Conference, Lecture Notes in Math., (1984).

[5] J. Hoffmann-Jørgensen, The Theory of Analytic Spaces, Various Publications Series No. 10, (1970), Aarhus Universitet, Aarhus.

[6] K. Kuratowski, Topology, Volume I (1966), Academic Press and PWN, New York,

[7] N. Lusin, Leçons sur les Ensembles Analytique, Gauthier-Villars (1930).

[8] (Szpilrajn), E. Marczewski, Sur une classe de fonctions de M. Sierpiński et la classe correspondante d'ensembles, Fund. Math., 24 (1935), 17-34.

[9] R. D. Mauldin, On non-isomorphic analytic sets, Proc. Amer. Math. Soc., 83 (1976), 369-370.

[10] S. Mazurkiewicz and W. Sierpiński, Sur une problème des fonctions continue, Fund. Math., 6 (1924), 161-169.

[11] B. V. Rao, K. P. S. Bhaskara Rao, Borel spaces, Dissertationes Mathematicae CXC (1981).

[12] R. M. Shortt, Borel-density, the marginal problem and isomorphism types of analytic sets, Pacific J. Math., 113 (1984), 183-200.

[13] _ Borel-dense Blackwell spaces are strongly Blackwell, (1983), (preprint).

Received October 6, 1983.

Michigan TeChNOLOGICAL UNIVERSITY

Houghton, MI 49931 


\section{PACIFIC JOURNAL OF MATHEMATICS EDITORS}

\author{
V. S. VARADARAJAN (Managing Editor) \\ University of California \\ Los Angeles, CA 90024 \\ Charles R. DePrima \\ California Institute of Technology \\ Pasadena, CA 91125 \\ R. FINN \\ Stanford University \\ Stanford, CA 94305
}

\author{
HeRmanN FlaschKa \\ University of Arizona \\ Tucson, AZ 85721 \\ Ramesh A. Gangolli \\ University of Washington \\ Seattle, WA 98195 \\ ROBION KIRBY \\ University of California \\ Berkeley, CA 94720
}

C. C. MOORE

University of California

Berkeley, CA 94720

H. SAMELSON

Stanford University

Stanford, CA 94305

HAROLD STARK

University of California, San Diego

La Jolla, CA 92093

ASSOCIATE EDITORS
R. ARENS
E. F. BECKENBACH
B. H. NeUmanN
F. WOLF
K. YosHIDA (1906-1982)

\section{SUPPORTING INSTITUTIONS}

UNIVERSITY OF ARIZONA

UNIVERSITY OF BRITISH COLUMBIA

CALIFORNIA INSTITUTE OF TECHNOLOGY

UNIVERSITY OF CALIFORNIA

MONTANA STATE UNIVERSITY

UNIVERSITY OF NEVADA, RENO

NEW MEXICO STATE UNIVERSITY

OREGON STATE UNIVERSITY
UNIVERSITY OF OREGON

UNIVERSITY OF SOUTHERN CALIFORNIA

STANFORD UNIVERSITY

UNIVERSITY OF HAWAII

UNIVERSITY OF TOKYO

UNIVERSITY OF UTAH

WASHINGTON STATE UNIVERSITY

UNIVERSITY OF WASHINGTON 


\section{Pacific Journal of Mathematics}

Vol. 119, No. $1 \quad$ May, 1985

Maurice Chacron, Nonisotropic unitary spaces and modules with

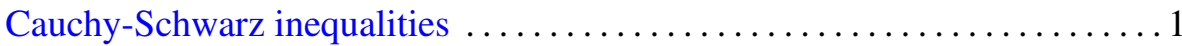

Myriam Dechamps-Gondim, Françoise Piquard and H. Queffélec, On

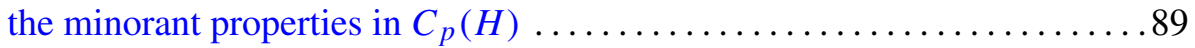

Klaus Floret and V. B. Moscatelli, On bases in strict inductive and projective limits of locally convex spaces .................... 103

Norman Joseph Goldstein, Degenerate secant varieties and a problem on

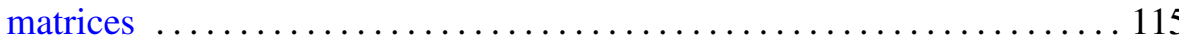

Harold Morris Hastings and Stefan Waner, $G$-bordism with singularities

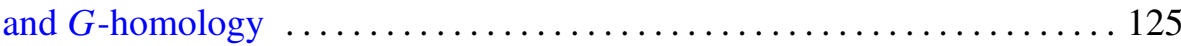

Takesi Isiwata, Clopen realcompactification of a mapping ........... 153

Hisao Kato, Concerning hyperspaces of certain Peano continua and strong

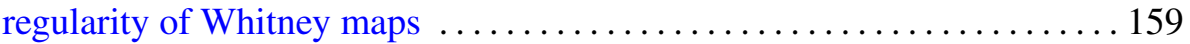

Elyahu Katz and Sidney Allen Morris, Free products of topological groups

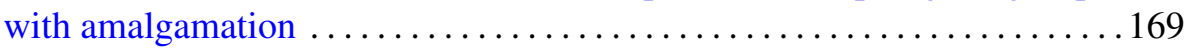

Kyewon Koh Park, Nice dense subsets for ergodic flows and Bernoulli

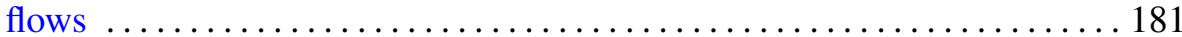

Francis Pastijn and Peter George Trotter, Lattices of completely regular

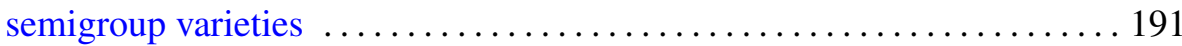

Rae Michael Andrew Shortt, Reticulated sets and the isomorphism of analytic powers

David A. Stegenga and Kenneth R. Stephenson, Generic covering properties for spaces of analytic functions

M. V. Subba Rao and R. Sitaramachandra Rao, On some infinite series of

L. J. Mordell and their analogues 